European Conference on Laboratory Astrophysics - ECLA

C. Stehlé, C. Joblin and L. d'Hendecourt (eds)

EAS Publications Series, 58 (2012) 113-116

www.eas.org

\title{
MHD SIMULATIONS OF JET-LAUNCHING FROM DIFFUSIVE MAGNETIZED ACCRETION DISKS
}

\author{
S. Sheikhnezami ${ }^{1,2}$ and C. Fendt ${ }^{1}$
}

\begin{abstract}
We investigate the launching process of jets from accretion disks by means of axisymmetric MHD simulations. Here, we present results of numerical MHD simulations investigating how magnetic diffusivity and numerical resolution may affect the ejection-to-accretion fraction and the asymptotic outflow structure.
\end{abstract}

\section{Introduction}

Astrophysical jets and outflows are a common phenomenon in the universe. They are observed over a wide range of luminosity and spatial scale - originating from young stellar objects (YSO), micro-quasars, active galactic nuclei (AGN), and most probably also in gamma ray bursts. Jets can be understood as channels along which mass, momentum, energy, and magnetic flux are transported through the ambient medium - either the interstellar or the intergalactic medium. The most powerful jets emerge from active galactic nuclei (AGNs) while the jets associated with low-mass young stellar objects have much lower energy.

The seminal model of jet formation applies steady-state MHD solutions for an axisymmetric, magneto-centrifugally driven outflow from a Keplerian disk threated by a large scale magnetic field Blandford \& Payne (1982). For the (cold) outflow to be launched, the poloidal magnetic field lines must be inclined less than $60^{\circ}$ with respect to the plane of the accretion disk.

This model has been further applied within the steady-state approach (Fendt \& Camenzind 1996; Pudritz \& Norman 1983), and simulations of jet-launching from the disk surface (Fendt \& Cemeljic 2002; Fendt 2006; Ouyed \& Pudritz 1997; Porth \& Fendt 2010), or considering also taking into account the disk evolution (Casse \& Keppens 2002; Zanni et al. 2007). Our simulation presented here also consider the latter approach, a disk wind accelerated by the magneto-centrifugal

1 Max-Planck-Institute for Astronomy, Königstuhl 17, 69117 Heidelberg, Germany;

e-mail: nezami@mpia.de; fendt@mpia.de

2 Department of Physics, Faculty of Sciences, Ferdowsi University of Mashhad, Iran 


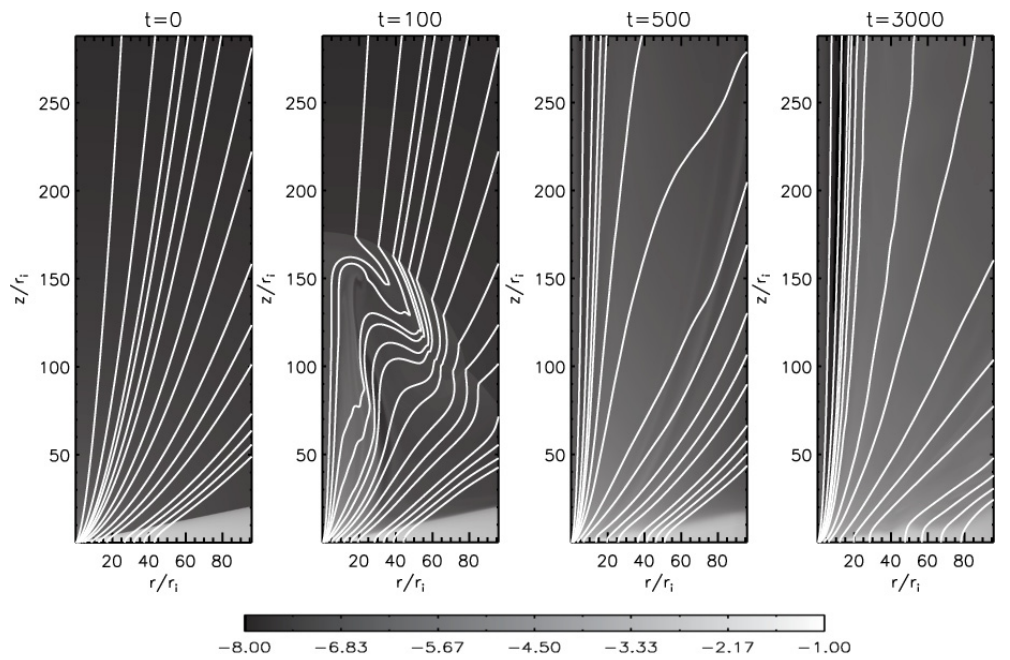

Fig. 1. Snapshots of MHD jet formation. Shown is the evolution of the mass density (color coding) and the poloidal magnetic field (contours of poloidal magnetic flux) for the dynamical time steps $t=0,500,1000,3000$.

driving mechanism, with material lifted from the disk midplane into the outflow. In particular, we present a time-dependent simulation of the outflow launching from a diffusive magnetized accretion disk for which we take into account the evolution of the underlying accretion process.

\section{Model setup}

We model the MHD jet launching from a slightly sub-Keplerian thin disk initially in pressure equilibrium with a non-rotating corona. The density contrast between the disk and corona is $\delta=10^{-4}$. We prescribe the initial configuration of accretion disk following the approach of Casse \& Keppens (2002) and Zanni et al. (2007). The magnetic diffusivity $\eta_{\mathrm{m}}$ plays an essential role in driving the accretion process. Turbulent magnetic diffusivity allows matter to steadily diffuse across the field line. The magnetic torque $F_{\phi}=J_{z} B_{r}-J_{r} B_{z}$ brakes the disk. The outflow carries out both the angular momentum and mechanical energy of the plasma (Ferreira \& Pelletier 1993). This is an efficient way of removing the angular momentum from the disk. Magnetic diffusivity allows for an equilibrium between advection of magnetic flux with the accretion flow and diffusive outwards. Inside the disk, we prescribe the anisotropic magnetic diffusivity as

$$
\eta_{\mathrm{m}}=\left.\alpha v_{\mathrm{A}}(r)\right|_{z=0} H(r) \exp \left(-2 \frac{z^{2}}{H_{\eta}(r)^{2}}\right),
$$

where $\alpha$ is diffusivity coefficient, $v_{\mathrm{A}}$ the initial Alfvén speed measured at the midplane, $H$ is the disk's thermal scale height, and $H_{\eta}$ is the scale height regarding 

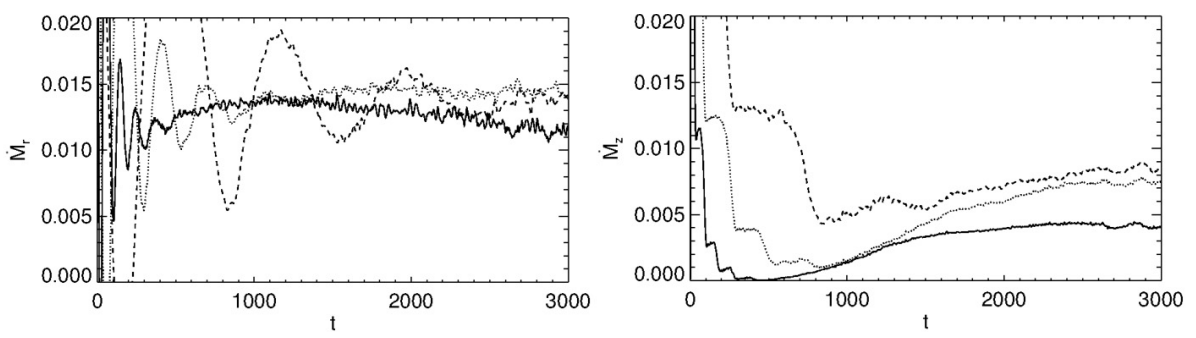

Fig. 2. Mass flux evolution. Shown is the evolution of the accretion rate (left panel) and ejection rate (right panel), for the presented simulation with $r 1=1, r 2=5$ (solid line), 10 (dotted line), 20 (dashed line).

the disk diffusivity. The initial magnetic field is prescribed by the magnetic flux function

$$
\psi(r, z)=\frac{3}{4} B_{z, 0} r_{0}^{2}\left(\frac{r}{r_{0}}\right)^{3 / 4} \frac{m^{5 / 4}}{\left(m^{2}+(z / r)^{2}\right)^{5 / 8}} .
$$

where $m$ determines field line inclination (Zanni et al. 2007). We solve the resistive MHD equations for the disk-jet structure using the MHD code PLUTO (Mignone et al. 2007). An internal boundary is imposed within the central part of the disk - a sink - to i) hide the singularity at the origin, and to ii) absorb the accreting plasma from the disk. At the outer boundary of the domain, we impose a modified outflow boundary condition (Porth \& Fendt 2010) to avoid any artificial collimation.

\section{Results and discussion}

Here we present results of simulations applying the following set of parameters, $m=0.4$, plasma- $\beta=20, \epsilon=H / r=0.1$. We observe a continuous and robust outflow formed from the inner part of the disk (Fig. 1). Our simulation reaches a quasi steady state at $t=3000$ and super-Alfvenic outflow velocities. In order to measure the mass flux loaded from the disk into the outflow, we adopt a control volume as a sector with two surfaces perpendicular to the equator at $r=r_{1}$ and $r=r_{2}$, and the two other surfaces being the disk mid plane and a surface which is parallel to the initial disk surface. Comparing the mass flux evolution in radial and vertical direction, we find an efficient accretion process in the disk (cf. Fig. 2). Depending on the radial position, a different period of time is required to establish dynamical equilibrium of inflow (accretion) and outflow (jet). Accretion establishes first at smaller radii and then spreads out to the larger radii. Higher accretion rates corresponding to larger radii imply that part of the accreting plasma is loaded into the outflow. About $30-40 \%$ of the accreting plasma is diverted into the outflow. We have also performed runs with different diffusivity scale height $H_{\eta}$. We find that this may affect the outflow structure considerably, as a lower diffusive scale height results in a more filamentary outflow. We also studied the aspect numerical resolution. We find that with increasing resolution, 


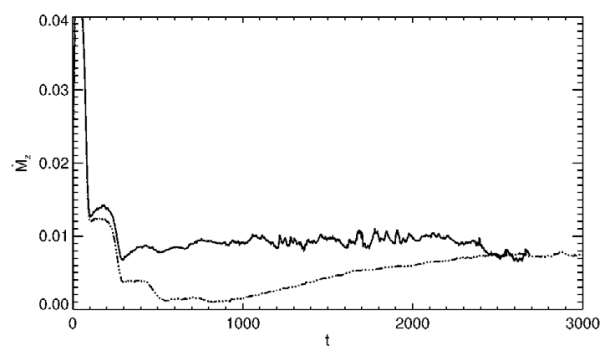

Fig. 3. Resolution comparison. Shown is the ejection rate evolution for presented simulation (solid line) and one with 3 times higher resolution (3dots-dashed line) for $r_{1}=1$ and $r_{2}=10$.

the ejection-to-the accretion ratio in general increases ( $c f$. Fig. 3), indicating a more efficient mass loading in the launching process. As for higher resolution thus decreasing numerical diffusivity - the coupling between the plasma and the field line decreases, the foot points of the field lines are further advected inward. The subsequent faster rotation induces a stronger $B_{\phi}$ leading to a stronger toroidal magnetic field pressure gradient, and finally a higher ejection rate.

We thank the SFB 881 for the financial support of this conference.

\section{References}

Blandford, R.D., \& Payne, D.G., 1982, MNRAS, 199, 883

Casse, F., \& Keppens, R., 2002, ApJ, 581, 988

Fendt, C., \& Camenzind, M., 1996, A\&A, 313, 591

Fendt, C., \& Cemeljic, M., 2002, A\&A, 395, 1045

Fendt, C., 2006, ApJ, 651, 272

Ferreira, J., \& Pelletier, G., 1993, A\&A, 276, 625

Mignone, A., Bodo, G., Massaglia, S., et al., 2007, ApJS, 170, 228

Ouyed, R., \& Pudritz, R.E., 1997, ApJ, 482, 712

Porth, O., \& Fendt, C., 2010, ApJ, 709, 1100

Pudritz, R.E., \& Norman, C.A., 1983, ApJ, 274, 677

Zanni, C., Ferrari, A., Rosner, R., et al., 2007, A\&A, 469, 811 